\title{
Measurements of $\mathrm{H}_{0}$ and reconstruction of the dark energy properties from a model-independent joint analysis
}

\author{
Alexander Bonilla ${ }^{1, a}$, Suresh Kumar ${ }^{2,3, b}$, Rafael C. Nunes ${ }^{4, c}$ \\ ${ }^{1}$ Departamento de Física, Universidade Federal de Juiz de Fora, Juiz de Fora, MG 36036-330, Brazil \\ 2 Department of Mathematics, National Institute of Technology, Kurukshetra, Haryana 136119, India \\ ${ }^{3}$ Department of Mathematics, BITS Pilani, Pilani Campus, Pilani, Rajasthan 333031, India \\ ${ }^{4}$ Divisão de Astrofísica, Instituto Nacional de Pesquisas Espaciais, Avenida dos Astronautas 1758, São José dos Campos, SP 12227-010, Brazil
}

Received: 23 December 2020 / Accepted: 26 January 2021 / Published online: 5 February 2021

(C) The Author(s) 2021

\begin{abstract}
Gaussian processes (GP) provide an elegant and model-independent method for extracting cosmological information from the observational data. In this work, we employ GP to perform a joint analysis by using the geometrical cosmological probes such as Supernova Type Ia (SN), Cosmic chronometers (CC), Baryon Acoustic Oscillations (BAO), and the HOLiCOW lenses sample to constrain the Hubble constant $H_{0}$, and reconstruct some properties of dark energy (DE), viz., the equation of state parameter $w$, the sound speed of DE perturbations $c_{s}^{2}$, and the ratio of DE density evolution $X=\rho_{\mathrm{de}} / \rho_{\mathrm{de}, 0}$. From the joint analysis $\mathrm{SN}+\mathrm{CC}+\mathrm{BAO}+\mathrm{H} 0 \mathrm{LiCOW}$, we find that $H_{0}$ is constrained at $1.1 \%$ precision with $H_{0}=73.78 \pm 0.84 \mathrm{~km} \mathrm{~s}^{-1} \mathrm{Mpc}^{-1}$, which is in agreement with SHOES and HOLiCOW estimates, but in $\sim 6.2 \sigma$ tension with the current $\mathrm{CMB}$ measurements of $H_{0}$. With regard to the DE parameters, we find $c_{s}^{2}<0$ at $\sim 2 \sigma$ at high $z$, and the possibility of $X$ to become negative for $z>1.5$. We compare our results with the ones obtained in the literature, and discuss the consequences of our main results on the DE theoretical framework.
\end{abstract}

\section{Introduction}

Several astronomical observations indicate that our Universe is currently in an accelerated expansion stage [1-5]. A cosmological scenario with cold dark matter (CDM) and dark energy (DE) mimicked by a positive cosmological constant, the so-called $\Lambda \mathrm{CDM}$ model, is considered the standard cosmological model, which fits the observational data with great precision. But, the cosmological constant suffers from some

\footnotetext{
a e-mail: abonilla@ fisica.ufjf.br

b e-mail: suresh.kumar@pilani.bits-pilani.ac.in

c e-mail: rafadcnunes@gmail.com (corresponding author)
}

theoretical problems [6-8], which motivate alternative considerations that can explain the data and have some theoretical appeal as well. In this regard, numerous cosmological models have been proposed in the literature, by introducing some new dark fluid with negative pressure or modification in the general relativity theory, where additional gravitational degree(s) can generate the accelerated stage of the Universe at late times (See [9-11] for a review). On the other hand, from an observational point of view, it is currently under discussion whether the $\Lambda \mathrm{CDM}$ model really is the best scenario to explain the observations, mainly in light of the current Hubble constant $H_{0}$ tension. Assuming the $\Lambda \mathrm{CDM}$ scenario, Planck-CMB data analysis provides $H_{0}=67.4 \pm 0.5 \mathrm{~km} \mathrm{~s}^{-1} \mathrm{Mpc}^{-1}$ [12], which is in $4.4 \sigma$ tension with a cosmological model-independent local measurement $H_{0}=74.03 \pm 1.42 \mathrm{~km} \mathrm{~s}^{-1} \mathrm{Mpc}^{-1}$ [13] from the Hubble Space Telescope (HST) observations of 70 long-period Cepheids in the Large Magellanic Cloud. Additionally, a combination of time-delay cosmography from HOLiCOW lenses and the distance ladder measurements are in $5.2 \sigma$ tension with the Planck-CMB constraints [14] (see also [15] for an update using HOLiCOW lens based new hierarchical approach where the mass-sheet transform is only constrained by stellar kinematics). Another accurate independent measure was carried out in [16], from Tip of the Red Giant Branch, obtaining $H_{0}=69.8 \pm 1.1 \mathrm{~km} \mathrm{~s}^{-1} \mathrm{Mpc}^{-1}$. Several other estimates of $H_{0}$ have been obtained in the recent literature (see [17-21]). It has been widely discussed in the literature whether a new physics beyond the standard cosmological model can solve the $H_{0}$ tension [22-36]. The so-called $S_{8}$ tension is also not less important. It is present between the Planck-CMB data with respect to weak lensing measurements and redshift surveys, about the value of the matter energy density $\Omega_{m}$ and the amplitude or growth rate of structures $\left(\sigma_{8}, f \sigma_{8}\right)$. We refer the reader to $[37,38]$ and references 
therein for perspectives and discussions on $S_{8}$ tension. Some other recent studies/developments [39-50] also suggest that the minimal $\Lambda C D M$ model is in crisis.

A promising approach for investigation of the cosmological parameters is to consider a model-independent analysis. In principle, this can be done via cosmographic approach [51-55], which consists of performing a series expansion of a cosmological observable around $z=0$, and then using the data to constrain the kinematic parameters. Such a procedure works well for lower values of $z$, but can be problematic at higher values of $z$. An interesting and robust alternative can be to consider a Gaussian process (GP) to reconstruct cosmological parameters in a model-independent way. The GP approach is a generic method of supervised learning (tasks to be learned and/or data training in GP terminology), which is implemented in regression problems and probabilistic classification. A GP is essentially a generalisation of the simple Gaussian distribution to the probability distributions of a function into the range of independent variables. In principle, this can be any stochastic process, however, it is much simpler in a Gaussian scenario and it is also more common, specifically for regression processes, which we use in this study. The GP also provides a model independent smoothing method that can further reconstruct derivatives from data. In this sense, the GP is a non-parametric strategy because it does not depend on a set of free parameters of the particular model to be constrained, although it depends on the choice of the covariance function, which will be explained in more detail in the next section. The GP method has been used to reconstruct the dynamics of the DE, modified gravity, cosmic curvature, estimates of Hubble constant, and other perspectives in cosmology by several authors [56-77].

In this work, our main aim is to employ GP to perform a joint analysis by using the geometrical cosmological probes such as Supernova Type Ia (SN), Cosmic chronometers (CC), Baryon Acoustic Oscillations (BAO), and the HOLiCOW lenses sample to constrain the Hubble constant $H_{0}$, and reconstruct some properties of $\mathrm{DE}$, viz., the equation of state parameter $w$, the sound speed of DE perturbations $c_{s}^{2}$, and the ratio of DE density evolution $X=\rho_{\mathrm{de}} / \rho_{\mathrm{de}, 0}$. These are the main quantities that can represent the physical characteristics of DE, and possible deviations from the standard values $w=-1, c_{s}^{2}=1$ and $X=1$, can be an indication of a new physics beyond the $\Lambda$ CDM model. To our knowledge, a model-independent joint analysis from above-mentioned data sets, as will be presented here, is new and not previously investigated in the literature. Indeed, a joint analysis with several observational probes is helpful to obtain tight constraints on the cosmological parameters.

This paper is structured as follows. In Sect. 2, we present the GP methodology as well as the data sets used in this work. In Sect. 3, we describe the modelling framework providing the cosmological information, and discuss our main results in detail. In Sect. 4, we summarize main findings of this study with some future perspectives.

\section{Methodology and data analysis}

In this section, we summarize our methodology as well as the data sets used for obtaining our results.

\subsection{Gaussian processes}

The main objective in a GP approximation is to reconstruct a function $f\left(x_{i}\right)$ from a set of its measured values $f\left(x_{i}\right) \pm \sigma_{i}$, where $x_{i}$ represent the training points or the positions of the observations. It assumes that the value of the function at any point $x_{i}$ follows a Gaussian distribution. The value of the function at $x_{i}$ is correlated with the value at other point $x_{i}^{\prime}$. Therefore, we may write the GP as

$f\left(x_{i}\right)=\mathcal{G P}\left(\mu\left(x_{i}\right), \operatorname{cov}\left[f\left(x_{i}\right), f\left(x_{i}\right)\right]\right)$,

where $\mu\left(x_{i}\right)$ and $\operatorname{cov}\left[f\left(x_{i}\right), f\left(x_{i}\right)\right]$ are the mean and the variance of the random variable at $x_{i}$, respectively. This method has been used in many studies in the context of cosmology (e.g. see [56-58]). For the reconstruction of the function $f\left(x_{i}\right)$, the covariance between the values of this function at different positions $x_{i}$ can be modeled as

$\operatorname{cov}\left[f(x), f\left(x^{\prime}\right)\right]=k\left(x, x^{\prime}\right)$,

where $k\left(x, x^{\prime}\right)$ is a priori assumed covariance model (or kernel in GP language), and its choice is often very crucial for obtaining good results regarding the reconstruction of the function $f\left(x_{i}\right)$. The covariance model, in general, depends on the distance $\left|x-x^{\prime}\right|$ between the input points $\left(x, x^{\prime}\right)$, and the covariance function $k\left(x, x^{\prime}\right)$ is expected to return large values when the input points $\left(x, x^{\prime}\right)$ are close to each other. The most popular and commonly used covariance functions in the literature are the standard Gaussian Squared-Exponential (SE) and the Matérn class of kernels $\left(M_{\nu}\right)$. The SE kernel is defined as

$k_{S E}\left(x, x^{\prime}\right)=\sigma_{f}^{2} \exp \left(-\frac{\left|x-x^{\prime}\right|^{2}}{2 l^{2}}\right)$

where $\sigma_{f}$ is the signal variance, which controls the strength of the correlation of the function, and $l$ is the length scale that determines the ability to model the main characteristics (global and local) in the evaluation region to be predicted (or coherence length of the correlation in $x$ ). These two parameters are often called hyperparameters. They are not the parameters of the function, but of the covariance function. For convenience, in what follows, we redefine $\tau=\left|x-x^{\prime}\right|$, which 
is consistent with all the kernels implemented here. The SE kernel, however, is a very smooth covariance function which can very well reproduce global but not local characteristics. To avoid this, the Matérn class kernels are helpful, and the general functional form can be written as

$k_{M_{\nu}}(\tau)=\sigma_{f}^{2} \frac{2^{1-v}}{\Gamma(v)}\left(\frac{\sqrt{2 v} \tau}{l}\right)^{v} K_{v}\left(\frac{\sqrt{2 v} \tau}{l}\right)$,

where $K_{v}$ is the modified Bessel function of second kind, $\Gamma(v)$ is the standard Gamma function and $v$ is strictly a positive parameter. An explicit analytic functional form for halfinteger values of $\{v=1 / 2,3 / 2,5 / 2,7 / 2,9 / 2, \ldots\}$ is provided by modified Bessel functions, and when $v \rightarrow \infty$, the $\mathrm{M}_{v}$ covariance function tends to SE kernel. Among other possibilities, $v=7 / 2$ and $v=9 / 2$ values are of primary interest, since these correspond to smooth functions with high predictability of derivatives of higher order, although these are not very suitable for predicting rapid variations. These Matern functions for GP in cosmology were first introduced in [58]. On the other hand, the hyperparameters $\Theta \equiv\left\{\sigma_{f}, l\right\}$ are learned by optimising the log marginal likelihood, which is defined as

$\mathcal{L}(\Theta)=-\frac{1}{2} \mathbf{y}^{\mathrm{T}} K_{\mathrm{y}}^{-1} \mathbf{y}-\frac{1}{2} \ln \left|K_{\mathrm{y}}\right|+\frac{n}{2} \ln (2 \pi)$,

where $K_{\mathrm{y}}=K\left(\mathbf{x}, \mathbf{x}^{\prime}\right)+C, K\left(\mathbf{x}, \mathbf{x}^{\prime}\right)$ is the covariance matrix with components $k\left(x_{i}, x_{j}\right), \mathbf{y}$ is the vector of data, $C$ is the covariance matrix of the data for a set of $n$ observations, assuming mean $\mu=0$. After optimizing for $\sigma_{f}$ and $l$, one can predict the mean and variance of the function $f\left(\mathbf{x}^{*}\right)$ at chosen points $\mathbf{x}^{*}$ through

$$
\begin{aligned}
\left\langle f\left(\mathbf{x}^{*}\right)\right\rangle & =K\left(\mathbf{x}^{*}, \mathbf{x}\right) K_{\mathrm{y}}^{-1} \mathbf{y} \\
\operatorname{cov}\left[f\left(\mathbf{x}^{*}\right)\right] & =K\left(\mathbf{x}^{*}, \mathbf{x}^{*}\right)-K\left(\mathbf{x}^{*}, \mathbf{x}\right) K_{\mathrm{y}}^{-1} K\left(\mathbf{x}, \mathbf{x}^{*}\right) .
\end{aligned}
$$

The GP predictions can also be extended to the derivatives of the functions $f\left(x_{i}\right)$, although limited by the differentiability of the chosen kernel. The derivative of a GP would also be a GP. Thus, one can obtain the covariance between the function and/or the derivatives involved by differentiating the covariance function as

$$
\begin{aligned}
\operatorname{cov}\left[f\left(x_{i}\right), \frac{\partial f\left(x_{j}\right)}{\partial x_{j}}\right] & =\frac{\partial k\left(x_{i}, x_{j}\right)}{\partial x_{j}} \\
\operatorname{cov}\left[\frac{\partial f\left(x_{i}\right)}{\partial x_{i}}, \frac{\partial f\left(x_{j}\right)}{\partial x_{j}}\right] & =\frac{\partial^{2} k\left(x_{i}, x_{j}\right)}{\partial x_{i} \partial x_{j}} .
\end{aligned}
$$

Then, we can write

$$
f^{\prime}\left(x_{i}\right)=\mathcal{G P}\left(\mu^{\prime}\left(x_{i}\right), \operatorname{cov}\left[\frac{\partial f\left(x_{i}\right)}{\partial x_{i}}, \frac{\partial f\left(x_{j}\right)}{\partial x_{j}}\right]\right),
$$

where $f^{\prime}\left(x_{i}\right)$ represent the derivatives with respect to their corresponding independent variables, which for our purpose can be the redshift $z$. This procedure can similarly be extended for higher derivatives $\left(f^{\prime}(x), f^{\prime \prime}(x), \ldots\right)$ in combination with $f(x)$. The mean of the $i^{t h}$ derivative and the covariance between $i^{\text {th }}$ and $j^{\text {th }}$ derivatives, are given by

$$
\begin{aligned}
\left\langle f^{(i)}\left(\mathbf{x}^{*}\right)\right\rangle & =K^{(i)}\left(\mathbf{x}^{*}, \mathbf{x}\right) K_{\mathrm{y}}^{-1} \mathbf{y} \\
\operatorname{cov}\left[f^{(i)}\left(\mathbf{x}^{*}\right), f^{(j)}\left(\mathbf{x}^{*}\right)\right]= & K^{(i, j)}\left(\mathbf{x}^{*}, \mathbf{x}^{*}\right) \\
& -K^{(i)}\left(\mathbf{x}^{*}, \mathbf{x}\right) K_{\mathrm{y}}^{-1} K^{(j)}\left(\mathbf{x}, \mathbf{x}^{*}\right) .
\end{aligned}
$$

If $i=j$, then we get the variance of the $i^{t h}$ derivative in Eq. (10). If the data for derivative functions are available, we can perform a joint analysis, which is the case in our study. Since one data type can be in terms of $f(x)$ while another can be rewritten in terms of $f^{\prime}(x)$, these different data sets can be combined. In what follows, we describe the data sets that we use in this work.

\subsection{Data sets}

We summarize below the data sets used in our analysis.

Cosmic chronometers (CC): The CC approach is a powerful method to trace the history of cosmic expansion through the measurement of $H(z)$. We consider the compilation of Hubble parameter measurements provided by [78]. This compilation consists of 30 measurements distributed over a redshift range $0<z<2$.

Baryon acoustic oscillations (BAO): The $\mathrm{BAO}$ is another important cosmological probe, which can trace expanding spherical wave of baryonic perturbations from acoustic oscillations at recombination time through the largescale structure correlation function, which displays a peak around $150 \mathrm{~h}^{-1} \mathrm{Mpc}$. We use BAO measurements from Sloan Digital Sky Survey (SDSS) III DR-12 at three effective binned redshifts $z=0.38,0.51$ and 0.61 , reported in [3], the clustering of the SDSS-IV extended Baryon Oscillation Spectroscopic Survey DR14 quasar sample at four effective binned redshifts $z=0.98,1.23,1,52$ and 1.94 , reported in [79], and the high-redshift Lyman- $\alpha$ measurements at $z=2.33$ and $z=2.4$ reported in [80] and [81], respectively. Note that the observations are presented in terms of $H(z) \times\left(r_{d} / r_{d}\right.$,fid $) \mathrm{km} \mathrm{s}^{-1} \mathrm{Mpc}^{-1}$, where $r_{d}$ is co-moving sound horizon and $r_{d, f i d}$ is the fiducial input value provided in the above references. In appendix A, we show that different $r_{d}$ input values obtained from different data sets do not affect the GP analysis.

Supernovae Type Ia (SN): The SN traditionally have been one of the most important astrophysical tools in establishing the so-called standard cosmological model. For the present analysis, we use the Pantheon compilation, which 

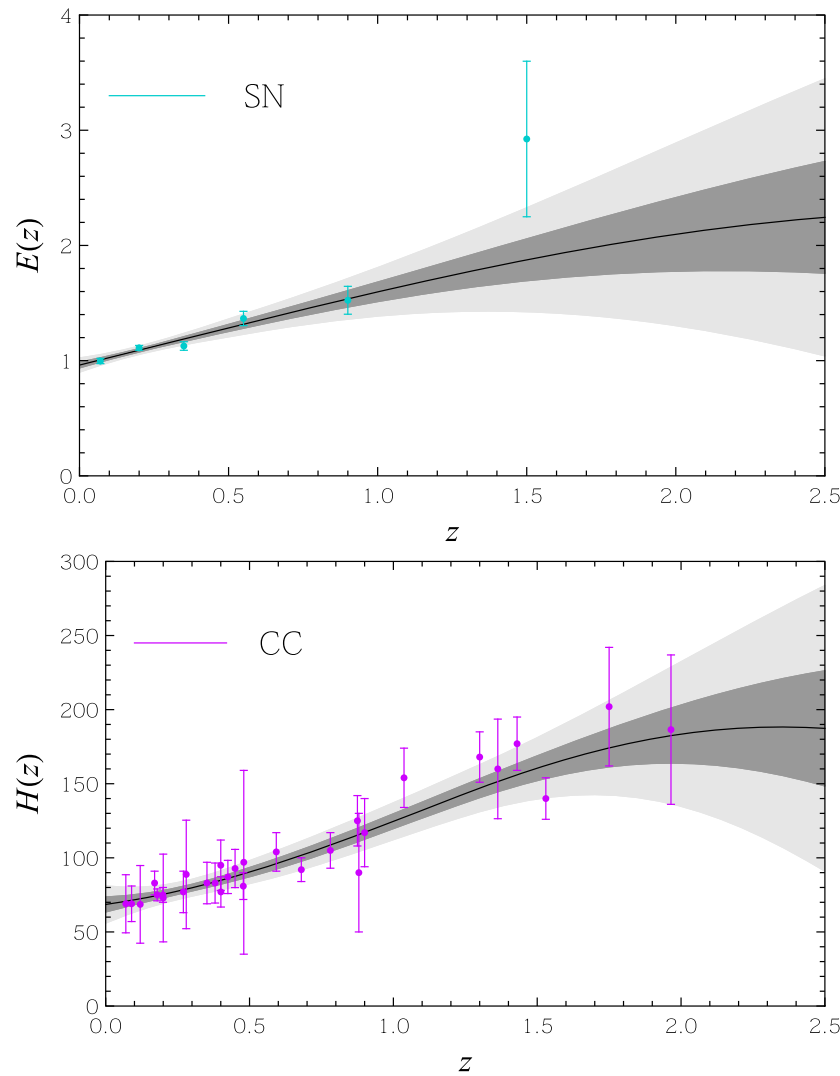

Fig. $1 H(z)$ (in units of $\mathrm{km} \mathrm{s}^{-1} \mathrm{Mpc}^{-1}$ ) vs $z(E(z)$ vs $z$ in case of $\mathrm{SN}$ data alone) with $1 \sigma$ and $2 \sigma \mathrm{CL}$ regions, reconstructed from SN (top-left), BAO (top-right), $\mathrm{CC}$ (bottom-left) and $\mathrm{SN}+\mathrm{BAO}+\mathrm{CC}$ data

consists of 1048 SNIa distributed in a redshift range $0.01<$ $z<2.3$ [82]. Under the consideration of a spatially flat Universe, the full sample of Pantheon can be summarized into six model independent $E(z)^{-1}$ data points [83]. We consider the six data points reported by [65] in the form of $E(z)$, including theoretical and statistical considerations made by the authors there for its implementation.

HOLiCOW sample: The Lenses in COSMOGRAIL's Wellspring program ${ }^{1}$ have measured six lens systems, making use of the measurements of time-delay distances between multiple images of strong gravitational lens systems by elliptical galaxies [14]. In the analyses of this work, we implement these six systems of strongly lensed quasars reported by the HOLiCOW Collaboration. Full information is contained in the so-called time-delay distance $D_{\Delta t}$. However, additional information can be found in the angular diameter distance to the lens $D_{l}$, which offers the possibility of using four additional data points in our analysis. Thus, our total HOLiCOW sample comprises of 10 data points: 6 measurements of timedelay distances and 4 angular diameter distances to the lens

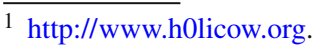
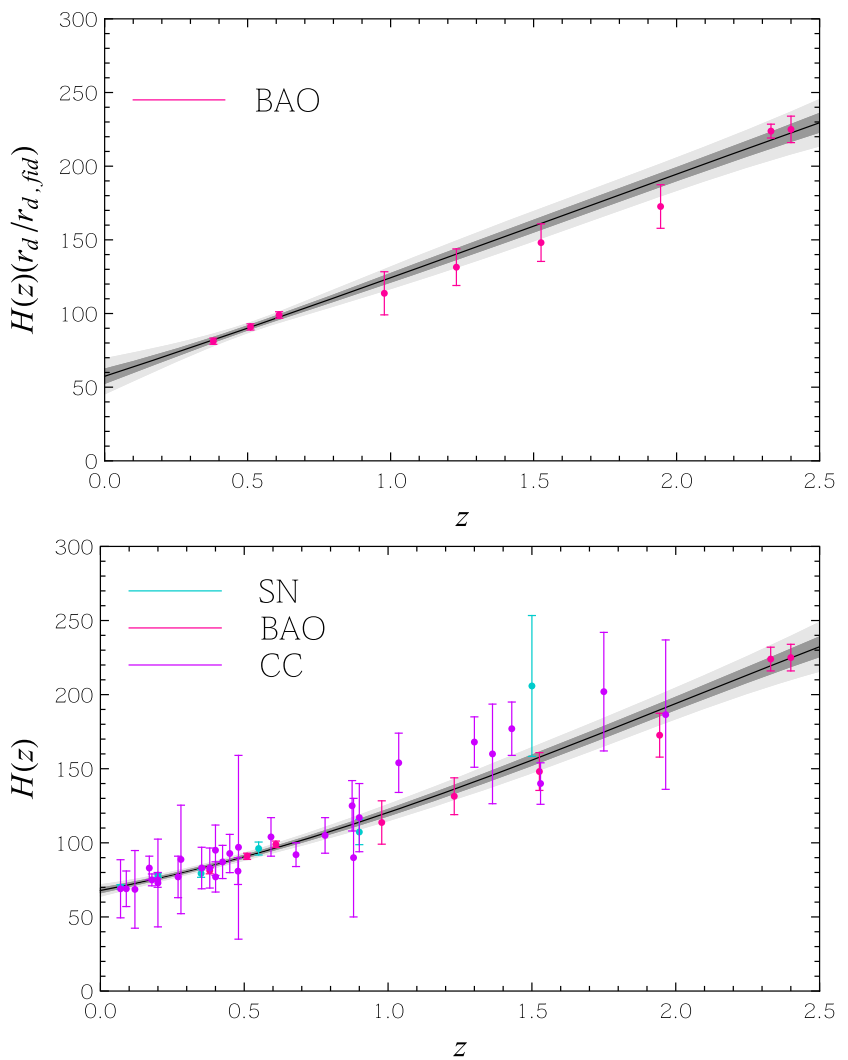

(bottom-right). Data with errorbars in all the panels are the observational data as mentioned in the legend of each panel

for 4 specific objects in the subset information in HOLiCOW sample (see [84,85] for the description).

\section{Results and discussions}

First, we verify that analyses carried out from $k_{M_{v}}(\tau)$, with $\tau=9 / 2$ and $\tau=7 / 2$, and $k_{S E}$ do not generate significantly different results, in the sense that all results are compatible with each other at $1 \sigma \mathrm{CL}$, and hence not generating any disagreement/tension between these input kernels. Thus, in what follows, we use GP formalism with an assumed $M_{9 / 2}$ kernel in the whole analysis. For this purpose, we have used some numerical routines available in the public GaPP code [56].

Figure 1 shows the reconstructions from $\mathrm{SN}, \mathrm{BAO}$ and CC data sets, using GP formalism on each data set individually. On the bottom-right panel, we show the $H(z)$ reconstruction from all these data together. First, from the $\mathrm{CC}$ reconstruction, we obtain $H_{0}=68.54 \pm$ $5.06 \mathrm{~km} \mathrm{~s}^{-1} \mathrm{Mpc}^{-1}$, which has been used in the rescaling process of $\mathrm{SN}$ data to carry out the joint analysis with 

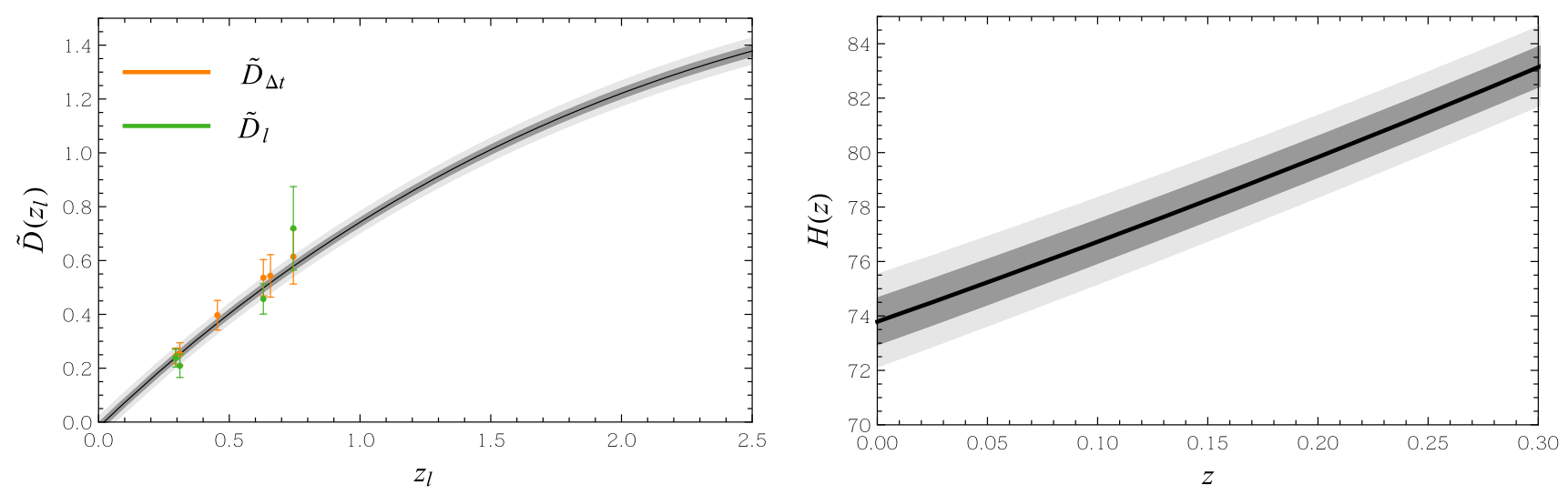

Fig. 2 Left panel: $\tilde{D}\left(z_{l}\right)$ vs $z_{l}$ with $1 \sigma$ and $2 \sigma$ CL regions, reconstructed from HOLiCOW sample data plus other data (SN+CC+BAO). Right panel: $H(z)$ vs $z$ with $1 \sigma$ and $2 \sigma \mathrm{CL}$ regions, enlarged in the redshift range $z<0.3$, and reconstructed from the combined data $\mathrm{SN}+\mathrm{BAO}+\mathrm{CC}+$ HOLiCOW

$\mathrm{SN}+\mathrm{BAO}+\mathrm{CC}$ (bottom-right). From $\mathrm{SN}+\mathrm{BAO}+\mathrm{CC}$ analysis, we find $H_{0}=67.85 \pm 1.53 \mathrm{~km} \mathrm{~s}^{-1} \mathrm{Mpc}^{-1}$. Figure 2 (left panel) shows the GP reconstruction of $D\left(z_{l}\right)$ from HOLiCOW data, where $z_{l}$ is the redshift to the lens. On the right panel, we show the reconstruction of $H(z)$ function from $\mathrm{SN}+\mathrm{BAO}+\mathrm{CC}+\mathrm{H} 0 \mathrm{LiCOW}$. In this joint analysis, we obtain $H_{0}=73.78 \pm 0.84 \mathrm{~km} \mathrm{~s}^{-1} \mathrm{Mpc}^{-1}$, which represents a $1.1 \%$ precision measurement. The strategy that we followed to obtain these results is as follows:

1. The $\mathrm{SN}+\mathrm{BAO}+\mathrm{CC}$ data set is used as in the previous joint analysis, i.e., in terms of $H(z)$ data reconstruction. Thus, now, we just need to re-scale the HOLiCOW data in some convenient way to combine all data for a joint analysis.

2. The time-delay distance in HOLiCOW sample is quantified as

$D_{\Delta t}=\left(1+z_{l}\right) \frac{D_{l} D_{s}}{D_{l s}}$

which is a combination of three angular diameter distances, namely $D_{l}, D_{s}$ and $D_{l s}$, where the subscripts stand for diameter distances to the lens $l$, to the source $s$, and between the lens and the source $l s$.

3. At this point, we can get the dimensionless co-moving distance through the relationship

$\tilde{D}(z)=\frac{H_{0}}{c}(1+z) D_{A}$,

where $D_{A}$ is the angular diameter distance and $\tilde{D}(z)$ is defined as $\tilde{D}(z)=\int_{0}^{z} \frac{d z^{\prime}}{E\left(z^{\prime}\right)}$. In this way, we can have: 6 data points from time delay distance $D_{\Delta t}$, which we referred to as $\tilde{D}_{\Delta t}$, and 4 data points obtained from angular diameter distance $D_{l}$, named as $\tilde{D}_{l}$. Thus, we can add these 10 data points for joint analysis, and name simply the HOLiCOW sample (see left panel of Fig. 2). Note that, to get $\tilde{D}_{l}$, we directly use the Eq. (12), where $D_{A}=D_{l}$. On the other hand, to obtain $\tilde{D}_{\Delta t}$, we have to take into account that Eq. (11) depends on the expansion rate of the Universe through $D_{s}\left(z_{s}, H_{0}, \Omega_{m}\right)$ and $D_{l s}\left(z_{l}, H_{0}, \Omega_{m}\right)$, and in this case, we use the $H_{0}$ and $\Omega_{m}$ best fit from our $\mathrm{SN}+\mathrm{BAO}+\mathrm{CC}$ joint analysis.

4. For the joint analysis, the relation $\tilde{D}(z)=\int_{0}^{z} \frac{d z^{\prime}}{E\left(z^{\prime}\right)}$ can be reversed to obtain $E(z)=\frac{1}{\tilde{D}^{\prime}(z)}$. So, we can make use of this possibility that offers the reconstruction of the first derivative of the dimensionless co-moving distance $\tilde{D}^{\prime}(z)$. For this purpose, we introduce the $\mathrm{SN}+\mathrm{BAO}+$ CC data set in the form of $1 / E(z)$ and the HOLiCOW data set in the form of $\tilde{D}(z)$, to obtain the GP reconstruction of dimensionless co-moving distance.

From the joint analysis $\mathrm{SN}+\mathrm{BAO}+\mathrm{CC}+\mathrm{HOLiCOW}$, we find $H(z=0)=73.78 \pm 0.84 \mathrm{~km} \mathrm{~s}^{-1} \mathrm{Mpc}^{-1}$. Figure 2 (right panel) shows the $H(z)$ reconstruction from $\mathrm{SN}+\mathrm{BAO}+\mathrm{CC}+\mathrm{H} 0 \mathrm{LiCOW}$. Figure 3 shows a comparison of our joint analysis estimates on $H_{0}$ with others recently obtained in literature. We note that our constraint on $H_{0}$ is in accordance with SHOES and HOLiCOW+STRIDES estimates. On the other hand, we find $\sim 6 \sigma$ tension with current Planck-CMB measurements and $\sim 2 \sigma$ tension with $\mathrm{CCHP}$ best fit. We re-analyze our estimates removing BAO data (see appendix A). In this case, we find $H_{0}=68.57 \pm$ $1.86 \mathrm{~km} \mathrm{~s}^{-1} \mathrm{Mpc}^{-1}$ and $H_{0}=71.65 \pm 1.09 \mathrm{~km} \mathrm{~s}^{-1} \mathrm{Mpc}^{-1}$ from $\mathrm{SN}+\mathrm{CC}$ and $\mathrm{SN}+\mathrm{CC}+\mathrm{HOLiCOW}$, respectively.

In the context of the standard framework, we can also check the $O_{m}(z)$ diagnostic [86]

$O_{m}(z)=\frac{E^{2}(z)-1}{(1+z)^{3}-1}$.

If the expansion history $E(z)$ is driven by the standard $\Lambda \mathrm{CDM}$ model, then $O_{m}(z)$ is practically constant and equal 


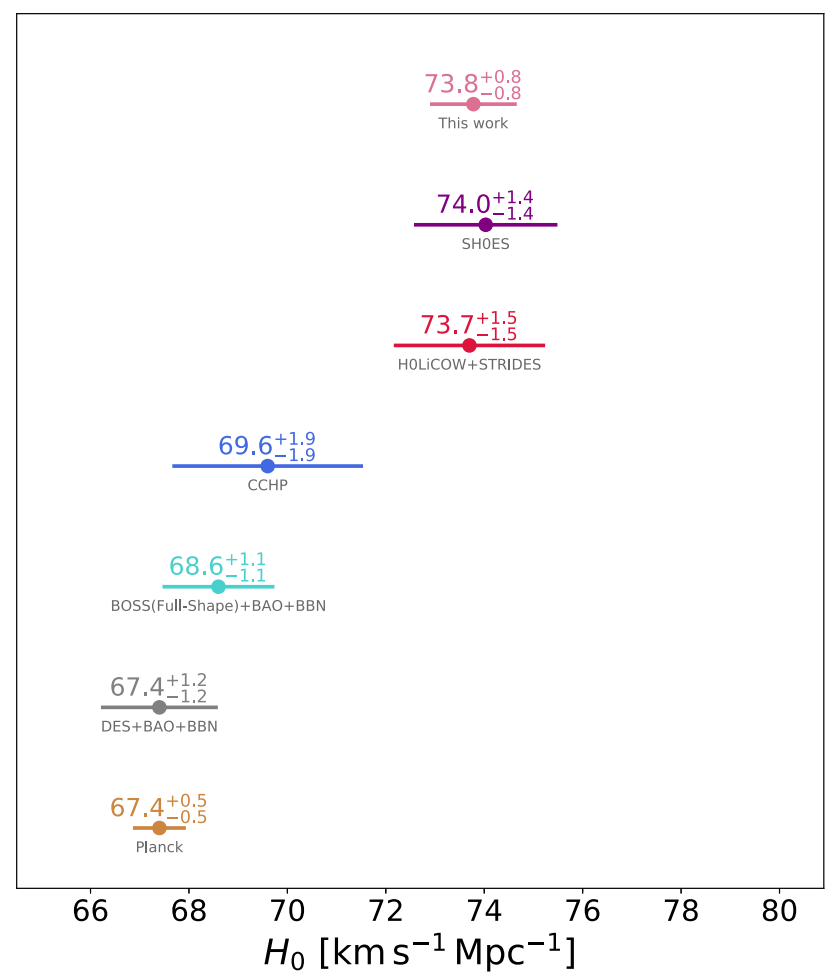

Fig. 3 Compilation of $H_{0}$ measurements taken from recent literature, namely, from Planck collaboration (Planck) [12], Dark Energy Survey Year 1 Results (DES+BAO+BBN) [118], the final data release of the BOSS data (BOSS Full-Shape+BAO+BBN) [117], The CarnegieChicago Hubble Program (CCHP) [16], HOLiCOW collaboration (HOLiCOW+STRIDES) [14], SHOES [13], in comparison with the $H_{0}$ constraints obtained in this work from the GP analysis using $\mathrm{SN}+\mathrm{BAO}+\mathrm{CC}+\mathrm{HOLiCOW}$

to the density of matter $\Omega_{m}$, and so, any deviation from this constant can be used to infer the dynamical nature of DE. Figure 4 shows the reconstruction of the $O_{m}(z)$ diagnostic. We find $\Omega_{m}=0.292 \pm 0.046$ and $\Omega_{m}=0.289 \pm 0.012$ at $1 \sigma$ from $\mathrm{SN}+\mathrm{BAO}+\mathrm{CC}$ and $\mathrm{SN}+\mathrm{BAO}+\mathrm{CC}+\mathrm{HOLiCOW}$ analyses, respectively. To obtain these results, we normal-

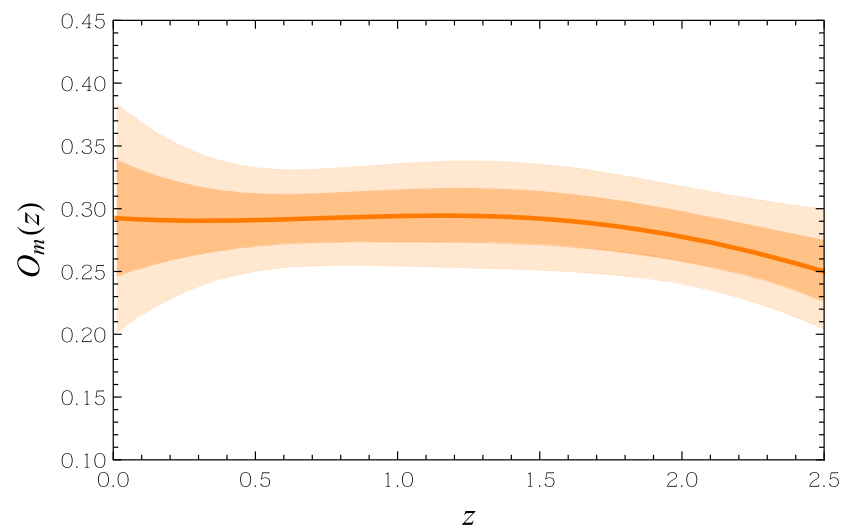

ize $H(z)$ with respect to $H_{0}$ to obtain $E(z)$ for the entire data set except $\mathrm{SN}$, where $H_{0}$ is taken from $\mathrm{SN}+\mathrm{BAO}+\mathrm{CC}$, and $\mathrm{SN}+\mathrm{BAO}+\mathrm{CC}+\mathrm{HOLiCOW}$ cases, respectively. The prediction from $\mathrm{SN}+\mathrm{BAO}+\mathrm{CC}$ is compatible with $\Omega_{m}=0.30$ across the analyzed range, but it is interesting to note that for $z>2$, we have $\Omega_{m}<0.30$ at $\sim 2 \sigma$ from $\mathrm{SN}+\mathrm{BAO}+\mathrm{CC}+\mathrm{H} 0 \mathrm{LiCOW}$. These model-independent $\Omega_{m}$ estimates will be used as input values in the reconstruction of $w$.

The EoS of DE can be written as [87-89]

$w(z)=\frac{2(1+z) E(z) E^{\prime}(z)-3 E^{2}(z)+\Omega_{k}(1+z)^{2}}{3\left(E^{2}(z)-\Omega_{m}(1+z)^{3}-\Omega_{k}(1+z)^{2}\right)}$,

where $\Omega_{m}$ and $\Omega_{k}$ are the density parameters of matter (baryonic matter + dark matter) and spatial curvature, respectively. In what follows, we assume $\Omega_{k}=0$, which is a strong, though quite general assumption about spatial geometry.

Figure 5 shows the $w(z)$ reconstruction from $\mathrm{SN}+\mathrm{BAO}+\mathrm{CC}$ and $\mathrm{SN}+\mathrm{BAO}+\mathrm{CC}+\mathrm{H} 0 \mathrm{LiCOW}$ data combinations on the left and right panels, respectively. From both analyses, we notice that $w$ is well constrained for $z \lesssim 0.5$ with the prediction $w=-1$. Most of the data correspond to this range in numbers and precision. The GP mean excludes any possibility of $w \neq-1$ in the whole range of $z$ under consideration. We observe that the best fit prediction is on $w=-1$ up to $z \sim 0.5$ for both cases. The addition of the HOLiCOW data considerably improve the reconstruction of $w$ for $z<1$. Beyond this range, the best fit prediction can deviate from $w=-1$, but statistically compatible with a cosmological constant. Evaluating at the present moment, we find $w(z=$ $0)=-0.999 \pm 0.093$ and $w(z=0)=-0.998 \pm 0.064$ from $\mathrm{SN}+\mathrm{BAO}+\mathrm{CC}$ and $\mathrm{SN}+\mathrm{BAO}+\mathrm{CC}+\mathrm{HOLiCOW}$, respectively. Note that HOLiCOW sample improves the constraints on $w(z=0)$ up to $\sim 2.9 \%$.

From the statistical reconstruction of $w(z)$ and its derivative $w^{\prime}(z)$, we can analyze the DE adiabatic sound speed $c_{s}^{2}$.

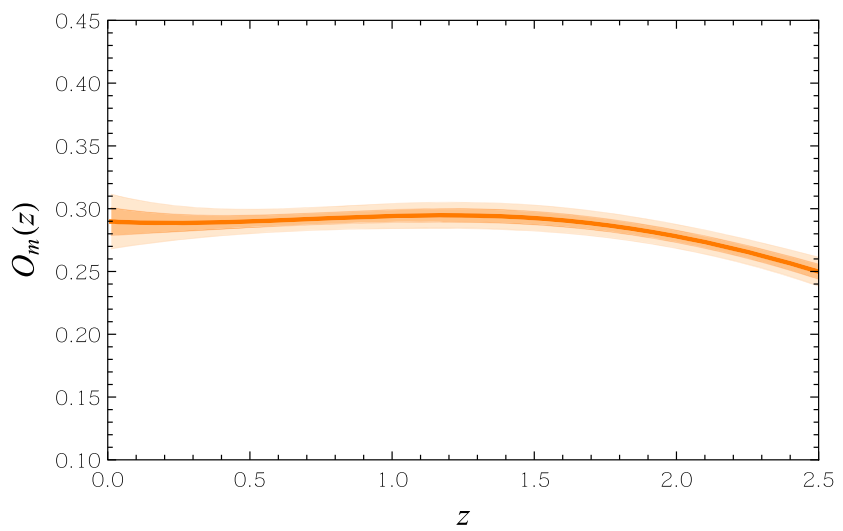

Fig. $4 O_{m}(z)$ vs $z$ with $1 \sigma$ and $2 \sigma \mathrm{CL}$ regions, reconstructed from $\mathrm{SN}+\mathrm{BAO}+\mathrm{CC}$ data (left panel) and $\mathrm{SN}+\mathrm{BAO}+\mathrm{CC}+\mathrm{H} 0 \mathrm{LiCOW}$ data (right panel) 

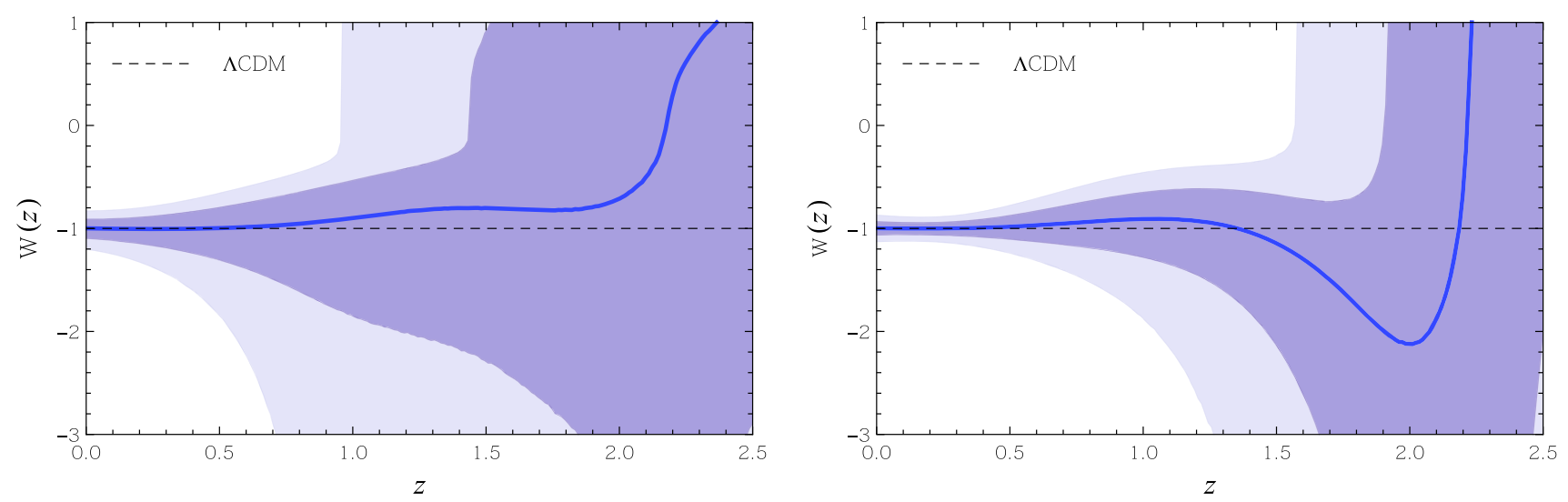

Fig. 5 The $\operatorname{EoS} w(z)$ vs $z$ with $1 \sigma$ and $2 \sigma \mathrm{CL}$ regions, reconstructed from $\mathrm{SN}+\mathrm{BAO}+\mathrm{CC}$ data (left panel) and $\mathrm{SN}+\mathrm{BAO}+\mathrm{CC}+\mathrm{H} 0 \mathrm{LiCOW}$ data (right panel)
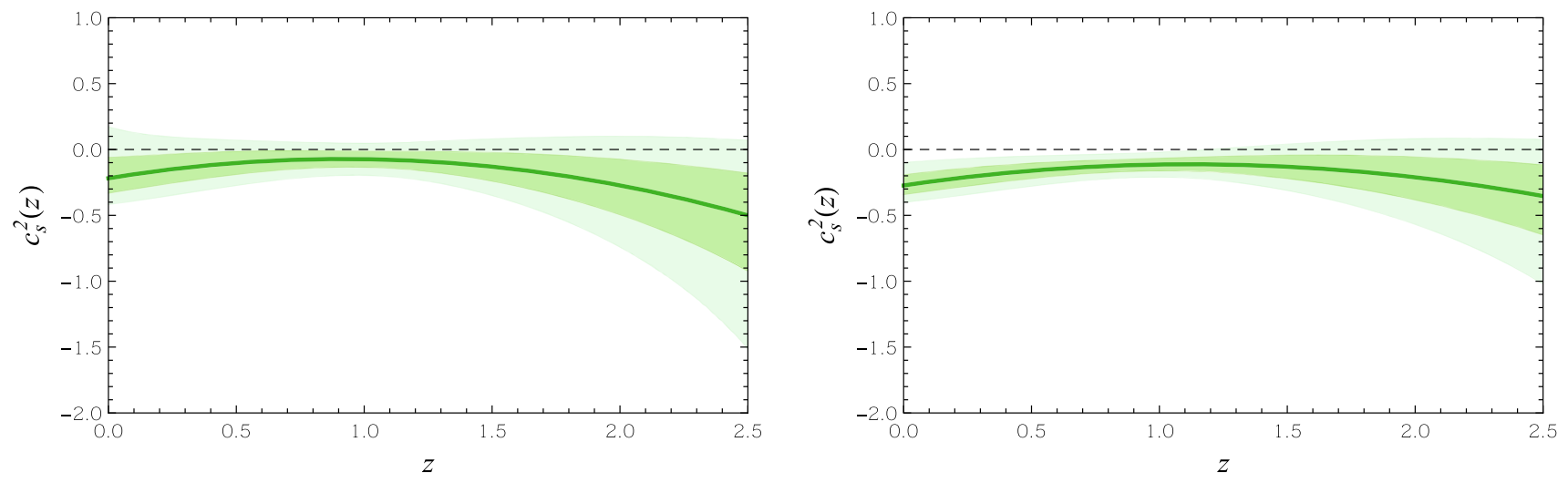

Fig. $6 c_{s}^{2}(z)$ vs $z$ with $1 \sigma$ and $2 \sigma \mathrm{CL}$ regions, reconstructed from $\mathrm{SN}+\mathrm{BAO}+\mathrm{CC}$ data (left panel) and $\mathrm{SN}+\mathrm{BAO}+\mathrm{CC}+\mathrm{H} 0 \mathrm{LiCOW}$ data (right panel). The dashed line is the division of dark energy clustering

Given the relation $p=w \rho$, we can find

$c_{s}^{2}(z)=\frac{\delta p}{\delta \rho}=w(z)+\frac{1+z}{3} \frac{w^{\prime}(z)}{1+w(z)}$.

Figure 6 shows $c_{s}^{2}$ reconstruction from $\mathrm{SN}+\mathrm{BAO}+\mathrm{CC}$ and $\mathrm{SN}+\mathrm{BAO}+\mathrm{CC}+\mathrm{H} 0 \mathrm{LiCOW}$ data combinations on the left and right panels, respectively. We note that the DE sound speed is negative at $\sim 1 \sigma$ from $\mathrm{SN}+\mathrm{BAO}+\mathrm{CC}$ when evaluated up to $z \simeq 2.5$. It is interesting to note that the $\mathrm{SN}+\mathrm{BAO}+\mathrm{CC}+\mathrm{H} 0 \mathrm{LiCOW}$ analysis yields $c_{s}^{2}<0$ at $2 \sigma$ for $z<1$. At the present moment, we find $c_{s}^{2}(z=0)=$ $-0.218 \pm 0.137$ and $c_{s}^{2}(z=0)=-0.273 \pm 0.068$ at $1 \sigma \mathrm{CL}$ from $\mathrm{SN}+\mathrm{BAO}+\mathrm{CC}$ and $\mathrm{SN}+\mathrm{BAO}+\mathrm{CC}+\mathrm{HOHiCOW}$, respectively. Therefore, this inference on $c_{s}^{2}$ rules out significantly the possibility for clustering DE models, and also the models with $c_{s}^{2}>0$ up to high $z$ at least at $1 \sigma \mathrm{CL}$. The condition $c_{s}^{2}>0$ is usually imposed to avoid gradient instability. However, the perturbations can still remain stable under $c_{s}^{2}<0$ consideration [92-95]. Thus, if the effective sound speed is negative, this would be a smoking gun signature for the existence of an anisotropic stress and possible modifications of gravity. Recently, a possible evidence for $c_{s}^{2}<0$ is found in [46], and also in a model-independent way from the Hubble data. Now, we look at some models which can potentially explain this result.

The Lagrangian $L=G_{2}(\phi, X)+\frac{M_{p l}^{2}}{2} R$ describes general $\mathrm{K}$-essence scenarios. Here the function $G_{2}$ depends on $\phi$ and $X=-\frac{1}{2} \nabla^{\mu} \phi \nabla_{\mu} \phi$, and $R$ is the Ricci scalar curvature. In this case, the sound speed is given by

$c_{s}^{2}=\frac{G_{2, X}}{G_{2, X}+\dot{\phi} G_{2, X X}}$,

where $G_{2, X} \equiv \partial G_{2} / \partial X$. Quintessence models correspond to the particular choice $G_{2}=X-V(\phi)$, given $c_{s}^{2}=1$. Thus, the usual quintessence scenarios are discarded from our results, which predict negative or low values of the sound speed.

Considering the so-called dilatonic ghost condensate [96], given by the Lagrangian,

$G_{2}=-X+e^{\lambda \phi / M_{p l}} \frac{X^{2}}{M^{4}}$ 

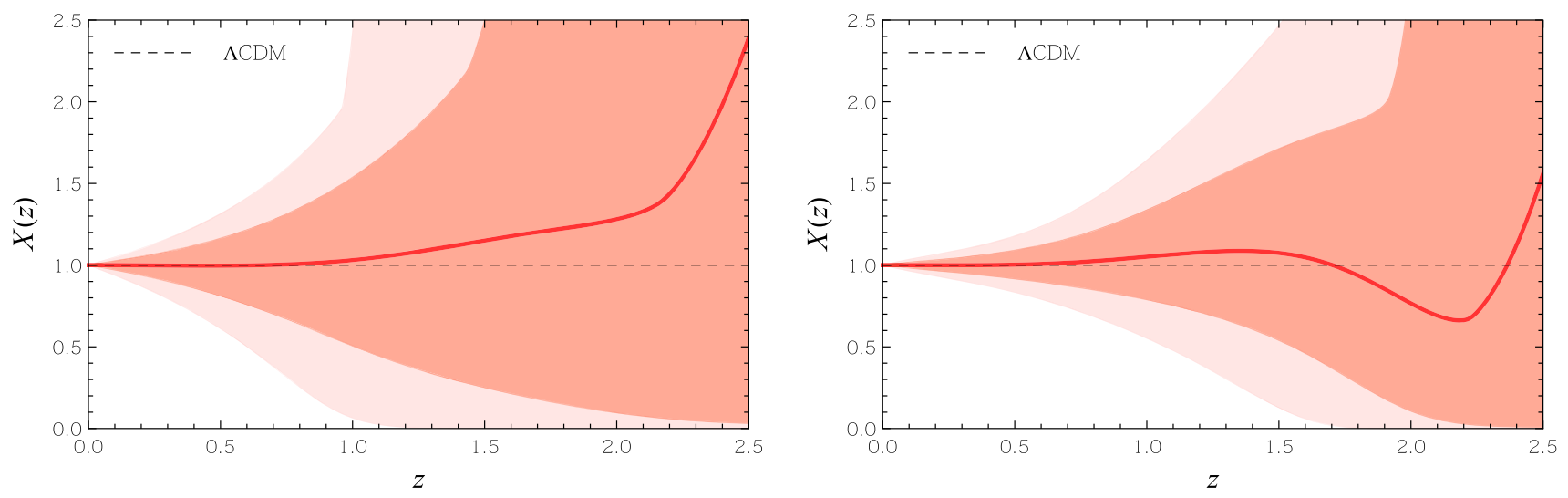

Fig. $7 X(z)$ vs $z$ with $1 \sigma$ and $2 \sigma \mathrm{CL}$ regions, reconstructed from $\mathrm{SN}+\mathrm{BAO}+\mathrm{CC}$ data (left panel) and $\mathrm{SN}+\mathrm{BAO}+\mathrm{CC}+\mathrm{H} 0 \mathrm{LiCOW}$ data (right panel). The dashed black curve corresponds to $\Lambda \mathrm{CDM}$ model prediction $X=1$

where $\lambda$ and $M$ are free parameters of the model, we can write $c_{s}^{2}$ as

$c_{s}^{2}=\frac{2 y-1}{6 y-1}$,

with $y=\frac{\dot{\phi}^{2} e^{\lambda \phi / M} p l}{2 M^{4}}$. The condition $y<-1 / 2$ ensures negative sound speed values.

Another interesting possibility pertains to a unified dark energy and dark matter scenario described by $G_{2}=-b_{0}+$ $b_{2}\left(X-X_{0}\right)^{2}$, where $b_{0}$ and $b_{2}$ are free parameters of the model [97]. In this case, the sound speed is

$c_{s}^{2}=\frac{X-X_{0}}{3 X-X_{0}}$,

where $c_{s}^{2}<0$ for $X<X_{0}$.

The above mentioned cases are theoretical examples under the consideration of a minimally coupled gravity scenario, which can reproduce a possible $c_{s}^{2}<0$ behavior. More generally, in the Horndeski theories of gravity [98-100], the speed of sound can be written as

$\alpha c_{s}^{2}=\left[\left(1-\frac{\alpha_{B}}{2}\right)\left(2 \alpha_{M}+\alpha_{B}\right)+\frac{\alpha_{B}}{2}\left(\ln H^{2}\right)^{\prime}+\alpha_{B}^{\prime}\right]$,

where prime denotes $d / d \ln a$, and $\alpha_{i}$ are functions expressed in a way that highlights their effects on the theory space [101], namely, kineticity $\left(\alpha_{K}\right)$, braiding $\left(\alpha_{B}\right)$ and Planck-mass run rate $\left(\alpha_{M}\right)$. Further, we define $\alpha=\alpha_{K}+3 / 2 \alpha_{B}^{2}$. Motivated for the tight constraints on the difference between the speed of gravitational waves and the speed of light to be $\lesssim 10^{-15}$ from the GW170817 and GRB 170817A observations [102,103], we assume $\alpha_{T}=0$ (tensor speed excess). Without loss of generality, we can consider $\alpha>0$ and the relation $\alpha_{B}=$ $R \times \alpha_{M}$, with $R$ being a constant. For instance, for $R=-1$, we reproduce $f(R)$ gravity theories. Different $R$ values can manifest the most diverse possible changes in gravity. For a qualitative example, taking $R=-1$, the running of the Planck mass must satisfy the relationship

$\frac{3}{2} \alpha_{M}^{2}-a \frac{d H}{d a} \frac{\alpha_{M}}{H}-a \frac{d \alpha_{M}}{d a} \leq 0$,

for generating $c_{s}^{2}<0$. At late cosmic time, we have $\frac{d H}{d a} \frac{1}{H}<$ 0 , and we can consider the theories in a good approximation where $\left|\alpha_{M} \ll 1\right|$. So we see that the condition $\alpha_{M}<0$, can generate negative $c_{s}^{2}$ values in this case.

Finally, we analyze the function

$X(z)=\frac{\rho_{\mathrm{de}}}{\rho_{\mathrm{de}, 0}}=\exp \left(3 \int_{0}^{z} \frac{1+w\left(z^{\prime}\right)}{1+z^{\prime}} d z^{\prime}\right)$,

quantifying the ratio of DE energy density evolution over the cosmic time.

Figure 7 shows $X(z)$ reconstruction from $\mathrm{SN}+\mathrm{BAO}+\mathrm{CC}$ and $\mathrm{SN}+\mathrm{BAO}+\mathrm{CC}+\mathrm{H} 0 \mathrm{LiCOW}$ data combinations on the left and right panels, respectively. We note that the evolution of $X$ is fully compatible with the $\Lambda \mathrm{CDM}$ model, and with the best fit model-independent prediction around $X=1$ up to $z \sim 1$, in both analyses. It is interesting to note that $X$ can cross to negative values when $z>1$ and $z>1.5$ at $2 \sigma \mathrm{CL}$ from $\mathrm{SN}+\mathrm{BAO}+\mathrm{CC}$ and $\mathrm{SN}+\mathrm{BAO}+\mathrm{CC}+\mathrm{H} 0 \mathrm{LiCOW}$, respectively. It can also have some interesting theoretical consequences. First, DE with negative density values at large $z$ came to the agenda when it turned out that, within the standard $\triangle \mathrm{CDM}$ model, the Ly- $\alpha$ forest measurement from BAO data by the BOSS collaboration [104], prefers a smaller value of the dust density parameter compared to the value preferred by the CMB data. Thus, with the possibility of a preference for negative energy density values at high $z$, it is argued that the Ly- $\alpha$ data at $z \sim 2.34$ can be described by a non-monotonic evolution in $H(z)$ function, which is difficult to achieve in any model with non-negative DE density [105]. Note that in our analysis, we are taking into account the high $z$ Lyman$\alpha$ measurements reported in [80] and [81]. It is possible to 
achieve $X<0$ at high $z$ when the cosmological gravitational coupling strength gets weaker with increasing $z[106,107]$. A range of other examples of effective sources crossing the energy density below zero also exists, including theories in which the cosmological constant relaxes from a large initial value via an adjustment mechanism [108], and also by modifying gravity theory [109-111]. More recently, a graduated DE model characterized by a minimal dynamical deviation from the null inertial mass density is introduced in [112] to obtain negative energy density at high $z$. Also, seeking inspiration from string theory, the possibility of negative energy density is investigated in [113].

The reconstruction of $w(z)$ and $X(z)$ are robust at low $z$, where the DE effects begin to be considerable, and a slow evolution of the EoS is well captured at $68 \%$ CL. However, the error estimates are larger at high $z$, where the data density is significantly smaller and the dynamical effects of DE are weaker. The introduction of the HOLiCOW data slightly improves the estimated errors in this range, especially for $1.0<z<1.5$. On the other hand, the uncertainties of smooth functions may have a greater amplitude than the highly oscillating functions, and in this way the propagation of errors to their derivatives can be overestimated [114]. In our case, the variation of the starting functions is quite smooth with respect to the data and their derivatives as well, leading to the propagation of errors with a greater amplitude, as can be seen in Figs. 5 and 7 at high $z$. Other aspects that may influence this fact could be the strong dependence on $z$, as in the case of $w(z)$, and the integrability of the functions with respect to $z$, as in the case of $X(z)$ (for a brief discussion in this regard, see for example [56]).

Recently, the authors in [115] have obtained a measurement $H_{0}=69.5 \pm 1.7 \mathrm{~km} \mathrm{~s}^{-1} \mathrm{Mpc}^{-1}$, showing that it is possible to constrain $H_{0}$ with an accuracy of $2 \%$ with minimal assumptions, from a combination of independent geometric datasets, namely, SN, BAO and CC. They have not used the HOLiCOW data in their analyses as we have used in the present work. They have also reconstructed the DE density parameter $X(z)$, finding similar conclusion as obtained here in this work.

\section{Final remarks}

We have applied GP to constrain $H_{0}$, and to reconstruct some functions that describe physical properties of DE in a modelindependent way using cosmological information from SN, $\mathrm{CC}, \mathrm{BAO}$ and $\mathrm{HOLiCOW}$ lenses data. The main results from the joint analysis, i.e., $\mathrm{SN}+\mathrm{CC}+\mathrm{BAO}+\mathrm{HOLiCOW}$, are summarized as follows: (i) A $1.1 \%$ accuracy measurement of $H_{0}$ is obtained with the best fit value $H_{0}=73.78 \pm 0.84 \mathrm{~km} \mathrm{~s}^{-1} \mathrm{Mpc}^{-1}$ at $1 \sigma \mathrm{CL}$.

(ii) The EoS of DE is measured at $\sim 6.5 \%$ accuracy at the present moment, with $w(z=0)=-0.98 \pm 0.064$ at $1 \sigma$ CL.

(iii) We find possible evidence for $c_{s}^{2}<0$ at $\sim 2 \sigma$ CL from the analysis of the function behavior at high $z$. At the present moment, we find $c_{s}^{2}(z=0)=-0.273 \pm 0.068$ at $1 \sigma \mathrm{CL}$

(iv) We find that the ratio of DE density evolution, $\rho_{\mathrm{de}} / \rho_{\mathrm{de}, 0}$, can cross to negative values at high- $z$. This behavior has already been observed by other authors. Here, we reconfirm this possibility for $z>1.5$ at $\sim 2 \sigma$.

Certainly, the GP method having the ability to perform joint analysis has a great potential in search for the accurate measurements of cosmological parameters, and analyze physical properties of the dark sector of the Universe in a minimally model-dependent way. It can shed light in the determination of the dynamics of the dark components or even rule out possible theoretical cosmological scenarios. Beyond the scope of the present work, it will be interesting to analyze/reconstruct a possible interaction in the dark sector, where DE and dark matter interact non-gravitationally in a model-independent way, through a robust joint analysis. Such scenarios have been intensively investigated recently in literature. We hope to communicate results in that direction in near future.

Acknowledgements The authors thank to Sunny Vagnozzi, Valerio Marra and Chris Clarkson for a critical reading of the manuscript and useful comments. S.K. gratefully acknowledges the support from SERB-DST project No. EMR/2016/000258. R.C.N. would like to thank the agency FAPESP for financial support under the project No. 2018/18036-5.

Data Availability Statement This manuscript has no associated data or the data will not be deposited. [Authors' comment: The data underlying this article will be shared on request to the corresponding author.]

Open Access This article is licensed under a Creative Commons Attribution 4.0 International License, which permits use, sharing, adaptation, distribution and reproduction in any medium or format, as long as you give appropriate credit to the original author(s) and the source, provide a link to the Creative Commons licence, and indicate if changes were made. The images or other third party material in this article are included in the article's Creative Commons licence, unless indicated otherwise in a credit line to the material. If material is not included in the article's Creative Commons licence and your intended use is not permitted by statutory regulation or exceeds the permitted use, you will need to obtain permission directly from the copyright holder. To view a copy of this licence, visit http://creativecomm ons.org/licenses/by/4.0/.

Funded by SCOAP 3 . 


\section{Appendix A: $H_{0}$ without BAO data, and effects of $r_{d}$}

In this appendix, we derive constraints on $H_{0}$ and $O_{m}(z)$ diagnostic removing our BAO data set compilation as described in Sect. II. Figure 8 shows $O_{m}(z)$ vs $z$ reconstructed from $\mathrm{SN}+\mathrm{CC}$ and $\mathrm{SN}+\mathrm{CC}+\mathrm{H} 0 \mathrm{LiCOW}$. For comparison, we also show the prediction with BAO. We find $H_{0}=68.57 \pm$ $1.86 \mathrm{~km} \mathrm{~s}^{-1} \mathrm{Mpc}^{-1}$ and $H_{0}=71.65 \pm 1.09 \mathrm{~km} \mathrm{~s}^{-1} \mathrm{Mpc}^{-1}$ from $\mathrm{SN}+\mathrm{CC}$ and $\mathrm{SN}+\mathrm{CC}+\mathrm{H} 0 \mathrm{LiCOW}$ data, respectively. Note that without BAO data these constraints are compatible with each other practically across the whole $z$ range under consideration, where the addition of the HOLiCOW sample, significantly improves the reconstruction compared to $\mathrm{SN}+\mathrm{CC}$. It is also interesting to observe the behavior for $z>1.5$, where we see that $\mathrm{Om}<0.31$. Combining $\mathrm{BAO}$ data with $\mathrm{SN}+\mathrm{CC}+\mathrm{HOLiCOW}$, we observe a significant improvement in the reconstruction for the whole $z$ range considered in the analysis. Predictions for $z>2$ disagree at $\sim 1.5 \sigma \mathrm{CL}$ when the GP mean is compared between $\mathrm{SN}+\mathrm{CC}+\mathrm{HOLiCOW}$ and $\mathrm{SN}+\mathrm{CC}+\mathrm{BAO}+\mathrm{H} 0 \mathrm{LiCOW}$.
On the other hand, the BAO measurements require a calibration of the sound horizon, either through BBN or the CMB. In all our analyses, we have used BAO data with the assumption $r_{d} / r_{d, f i d}=1$, where $r_{d, f i d}$ is the fiducial input value. In order to quantify how much the $r_{d}$ value can influence the GP reconstruction, we have analyzed $\operatorname{Om}(z)$ with different $r_{d}$ input values. We have used $r_{d}$ values obtained from Planck-CMB data [12] and eBOSS Collaboration [116]. Figure 9 shows $O_{m}(z)$, reconstructed using $r_{d}=149.30 \mathrm{Mpc}$ (eBOSS estimation) and $r_{d}=147.09 \mathrm{Mpc}$ (Planck-CMB estimation). In short, we conclude that appropriate and different $r_{d}$ input values do not change the results significantly in all the analyses carried out in this work. Any input value of $r_{d} \in[135,155] \mathrm{Mpc}$ does not have statistical divergence compared to assumption $r_{d} / r_{d, f i d}=1$. That is, all analyses are consistent with each other at $<1 \sigma$. Therefore, the GP analyses here are not sensitive to $r_{d}$. That is why, we have presented the results in the main text assuming $r_{d} / r_{d, f i d}=1$.
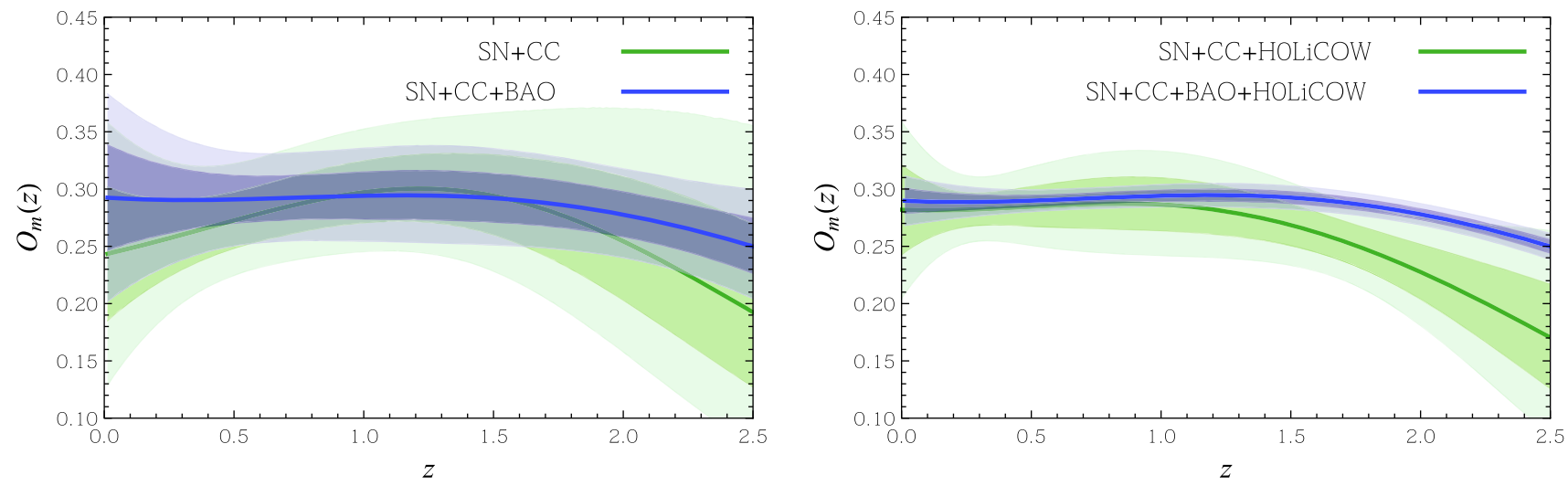

Fig. $8 O_{m}(z)$ vs $z$ with $1 \sigma$ and $2 \sigma \mathrm{CL}$ regions, reconstructed from $\mathrm{SN}+\mathrm{CC}$ (green) and $\mathrm{SN}+\mathrm{CC}+\mathrm{BAO}$ (blue) in the left panel, and $\mathrm{SN}+\mathrm{CC}+\mathrm{HOLiCOW}$ (green) and $\mathrm{SN}+\mathrm{CC}+\mathrm{HOLiCOW}+\mathrm{BAO}$ (blue) in the right panel
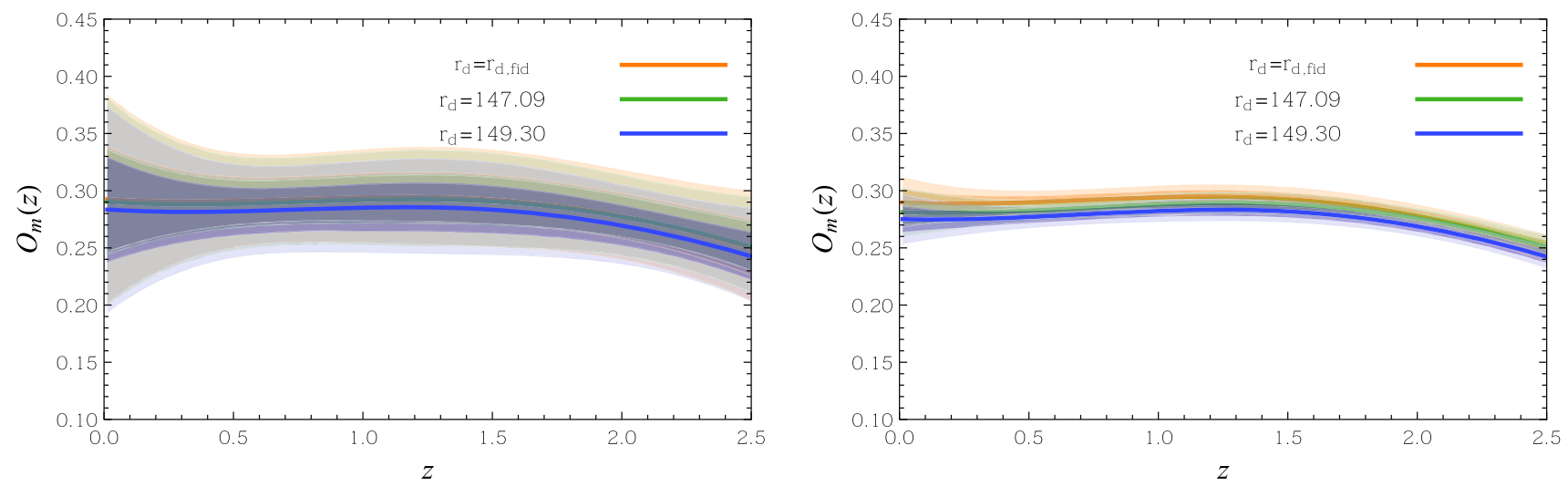

Fig. $9 O_{m}(z)$ vs $z$ with $1 \sigma$ and $2 \sigma \mathrm{CL}$ regions for different values of $r_{d}$ (in units of Mpc), reconstructed from $\mathrm{SN}+\mathrm{BAO}+\mathrm{CC}$ data (left panel) and $\mathrm{SN}+\mathrm{BAO}+\mathrm{CC}+\mathrm{H} 0 \mathrm{LiCOW}$ data (right panel) 


\section{References}

1. A.G. Riess et al., Observational evidence from supernovae for an accelerating universe and a cosmological constant. Astron. J. 116, 1009 (1998). arXiv:astro-ph/9805201

2. S. Perlmutter et al., [Supernova Cosmology Project], Measurements of $\Omega$ and $\Lambda$ from 42 high-redshift supernovae. Astrophys. J. 517, 565 (1999). arXiv:astro-ph/9812133

3. S. Alam et al., [BOSS], The clustering of galaxies in the completed SDSS-III Baryon Oscillation Spectroscopic Survey: cosmological analysis of the DR12 galaxy sample. Mon. Not. Roy. Astron. Soc. 470, 2617 (2017). arXiv:1607.03155

4. T. Abbott et al., [DES], Dark Energy Survey year 1 results: Cosmological constraints from galaxy clustering and weak lensing. Phys. Rev. D 98, 043526 (2018). arXiv: 1708.01530

5. S. Nadathur, W.J. Percival, F. Beutler, H. Winther, Testing lowredshift cosmic acceleration with large-scale structure. Phys. Rev. Lett. 124, 221301 (2020). arXiv:2001.11044

6. S. Weinberg, The cosmological constant problem. Rev. Mod. Phys. 61, 1 (1989)

7. T. Padmanabhan, Cosmological constant: the weight of the vacuum. Phys. Rept. 380, 235 (2003). arXiv:hep-th/0212290

8. R. Bousso, TASI lectures on the cosmological constant. Gen. Relat. Gravit. 40, 607 (2008). arXiv:0708.4231

9. D. Huterer, D.L. Shafer, Dark energy two decades after: observables, probes, consistency tests. Rep. Prog. Phys. 81, 016901 (2017). arXiv: 1709.01091

10. S. Capozziello, R. D'Agostino, O. Luongo, Extended gravity cosmography. Int. J Mod. Phys. D 28, 1930016 (2019). arXiv: 1904.01427

11. M. Ishak, Testing general relativity in cosmology. Living Rev. Rel. 1, 22 (2019). arXiv:1806.10122

12. N. Aghanim et al. (Planck Collaboration), Planck 2018 results. VI. Cosmological parameters. arXiv:1807.06209

13. A.G. Riess, S. Casertano, W. Yuan, L.M. Macri, D. Scolnic, Large magellanic cloud cepheid standards provide a $1 \%$ foundation for the determination of the hubble constant and stronger evidence for physics beyond $\Lambda$ CDM. Astrophys. J. 876, 1 (2019). arXiv: 1903.07603

14. K.C. Wong et al., (HOLiCOW Collaboration), HOLiCOW XIII. A $2.4 \%$ measurement of $\mathrm{H} 0$ from lensed quasars: $5.3 \sigma$ tension between early and late-Universe probes. arXiv:1907.04869

15. S. Birrer et al., TDCOSMO IV: Hierarchical time-delay cosmography - joint inference of the Hubble constant and galaxy density profiles. arXiv:2007.02941

16. W.L. Freedman et al., The Carnegie-Chicago Hubble Program. VIII. An independent determination of the hubble constant based on the tip of the red giant branch. arXiv:1907.05922

17. D. Camarena, V. Marra, Local determination of the Hubble constant and the deceleration parameter. Phys. Rev. Res. 2, 013028 (2020). arXiv: 1906.11814

18. D. Camarena, V. Marra, A new method to build the (inverse) distance ladder. MNRAS 495, 3 (2020). arXiv: 1910.14125

19. R.C. Nunes, A. Bernui, $\theta_{B A O}$ estimates and the $H_{0}$ tension, arXiv:2008.03259

20. N. Schoneberg, J. Lesgourgues, D.C. Hooper, The BAO+BBN take on the Hubble tension. J. Cosmol. Astrop. Phys. 10, 029 (2019). arXiv: 1907.11594

21. O.H.E. Philcox, B.D. Sherwin, G.S. Farren, E.J. Baxter, Determining the Hubble Constant without the Sound Horizon: Measurements from Galaxy Surveys. arXiv:2008.08084

22. L. Verde, T. Treu, A.G. Riess, Tensions between the early and late Universe. Nat. Astron. 3, 891 (2019). arXiv:1907.10625
23. V. Poulin, T.L. Smith, T. Karwal, M. Kamionkowski, Early dark energy can resolve the hubble tension. Phys. Rev. Lett. 122, 221301 (2019). arXiv:1811.04083

24. E. Mörtsell, S. Dhawan, Does the Hubble constant tension call for new physics? J. Cosmol. Astrop. Phys. 09, 025 (2018). arXiv: 1801.07260

25. R.C. Nunes, Structure formation in $f(T)$ gravity and a solution for $H_{0}$ tension. J. Cosmol. Astrop. Phys. 05, 052 (2018). arXiv: 1802.02281

26. W. Yang et al., Tale of stable interacting dark energy, observational signatures, and the $H_{0}$ tension. J. Cosmol. Astrop. Phys. 09, 019 (2018). arXiv:1805.08252

27. S. Pan, W. Yang, E. Di Valentino, A. Shafieloo, S. Chakraborty, Reconciling $H_{0}$ tension in a six parameter space? arXiv: 1907.12551

28. S. Kumar, R.C. Nunes, S.K. Yadav, Dark sector interaction: a remedy of the tensions between CMB and LSS data. Eur. Phys. J. C 79, 576 (2019). arXiv:1903.04865

29. E. Di Valentino, A. Melchiorri, O. Mena, S. Vagnozzi, Interacting dark energy after the latest Planck, DES, and $H_{0}$ measurements: an excellent solution to the $\mathrm{H} 0$ and cosmic shear tensions. arXiv: 1908.04281

30. S. Vagnozzi, New physics in light of the $\mathrm{H} 0$ tension: an alternative view. arXiv: 1907.07569

31. R. D'Agostino, R.C. Nunes, Measurements of $H_{0}$ in modified gravity theories: the role of lensed quasars in the late-time Universe. Phys. Rev. D 101, 103505 (2020). arXiv:2002.06381

32. S. Vagnozzi, E. Di Valentino, S. Gariazzo, A. Melchiorri, O. Mena, J. Silk, Listening to the BOSS: the galaxy power spectrum take on spatial curvature and cosmic concordance. arXiv:2010.02230

33. B.S. Haridasu, M. Viel, Late-time decaying dark matter: constraints and implications for the H0-tension. Mon. Not. Roy. Astron. Soc. 497, 2 (2020). arXiv:2004.07709

34. E. Di Valentino, A (brave) combined analysis of the $H_{0}$ late time direct measurements and the impact on the Dark Energy sector. arXiv:2011.00246

35. E. Di Valentino, A. Melchiorri, O. Mena, S. Pan, W. Yang, Interacting Dark Energy in a closed universe. arXiv:2011.00283

36. S. Pan, W. Yang, C. Singha, E.N. Saridakis, Observational constraints on sign-changeable interaction models and alleviation of the $H_{0}$ tension. Phys. Rev. D 100(8), 083539 (2019). arXiv: 1903.10969

37. A. Bonilla Rivera, J. García Farieta, Exploring the dark universe: constraints on dynamical dark energy models from CMB, $\mathrm{BAO}$ and growth rate measurements. Int. J Mod. Phys. D 28(09), 1950118 (2019). arXiv: 1605.01984

38. Eleonora Di Valentino et al., Cosmology Intertwined III: $f \sigma_{8}$ and $S_{8}$. arXiv:2008.11285

39. E. Di Valentino, A. Melchiorri, J Silk, Cosmic discordance: planck and luminosity distance data exclude LCDM. arXiv:2003.04935

40. E. Di Valentino, A. Melchiorri, J. Silk, Planck evidence for a closed Universe and a possible crisis for cosmology. Nat. Astron. 4, 196 (2020). arXiv: 1911.02087

41. S. Kumar, R.C. Nunes, Probing the interaction between dark matter and dark energy in the presence of massive neutrinos. Phys. Rev. D 94, 123511 (2016). arXiv: 1608.02454

42. S. Kumar, and R.C. Nunes, Echo of interactions in the dark sector. Phys. Rev. D 96, 103511 (2017). arXiv:1702.02143

43. G.B. Zhao et al., Dynamical dark energy in light of the latest observations. Nat. Astron. 1, 627 (2017). arXiv:1701.08165

44. S. Peirone, G. Benevento, N. Frusciante, S. Tsujikawa, Cosmological data favor Galileon ghost condensate over $\Lambda$ CDM. Phys. Rev. D 100, 063540 (2019). arXiv:1905.05166

45. A. Chudaykin, D. Gorbunov, N. Nedelko, Combined analysis of Planck and SPTPol data favors the early dark energy models. arXiv:2004.13046 
46. R. Arjona, S. Nesseris, Hints of dark energy anisotropic stress using Machine Learning. arXiv:2001.11420

47. L. Kazantzidis, L. Perivolaropoulos, Is gravity getting weaker at low z? Observational evidence and theoretical implications. arXiv: 1907.03176

48. E. Di Valentino, A. Melchiorri, J. Silk, Cosmological hints of modified gravity? Phys. Rev. D 93, 023513 (2016). arXiv:1509.07501

49. S. Pan, W. Yang, A. Paliathanasis, Non-linear interacting cosmological models after Planck legacy release and the $H_{0}$ tension. Mon. Not. Roy. Astron. Soc. 493(3), 3114-3131 (2018). arXiv:2002.03408

50. D. Benisty, Quantifying the $S_{8}$ tension with the Redshift Space Distortion data set. Phys. Dark Univ. 31, 100766 (2021). arXiv:2005.03751

51. C. Cattoen, M. Visser, Cosmographic Hubble fits to the supernova data. Phys. Rev. D 78, 063501 (2008). arXiv:0809.0537

52. S. Capozziello, R. D'Agostino, O. Luongo, Cosmographic analysis with Chebyshev polynomials. Mon. Not. Roy. Astron. Soc. 476, 3924 (2018). arXiv: 1712.04380

53. C. Cattoen, M. Visser, The Hubble series: Convergence properties and redshift variables. Class. Quant. Grav. 24, 5985 (2007). arXiv:0710.1887

54. E.M. Barboza Jr., F. Carvalho, A kinematic method to probe cosmic acceleration. Phys. Lett. B 715, 19 (2012)

55. C. Rodrigues Filho, E.M. Barboza, Constraints on kinematic parameters at $z \neq 0$ 0. J. Cosmol. Astrop. Phys. 07, 037 (2018). arXiv: 1704.08089

56. M. Seikel, C. Clarkson, M. Smith, Reconstruction of dark energy and expansion dynamics using Gaussian processes. J. Cosmol. Astrop. Phys. 07, 036 (2012). arXiv: 1204.2832

57. A. Shafieloo, A.G. Kim, E.V. Linder, Gaussian process cosmography. Phys. Rev. D 85, 123530 (2012). arXiv:1204.2272

58. M. Seikel, C. Clarkson, Optimising Gaussian processes for reconstructing dark energy dynamics from supernovae. arXiv: 1311.6678

59. M.J. Zhang, J.Q. Xia, Test of the cosmic evolution using Gaussian processes. J. Cosmol. Astrop. Phys. 12, 005 (2016). arXiv: 1606.04398

60. V.C. Busti, C. Clarkson, M. Seikel, Evidence for a Lower Value for $H_{0}$ from Cosmic Chronometers Data? Mon. Not. Roy. Astron. Soc. 441, 11 (2014). arXiv: 1402.5429

61. V. Sahni, A. Shafieloo, A.A. Starobinsky, Model independent evidence for dark energy evolution from Baryon Acoustic Oscillations. Astrophys. J. Lett. 793, L40 (2014). arXiv:1406.2209

62. E. Belgacem, S. Foffa, M. Maggiore, T. Yang, Gaussian processes reconstruction of modified gravitational wave propagation. Phys. Rev. D 101, 063505 (2020). arXiv:1911.11497

63. A.M. Pinho, S. Casas, L. Amendola, Model-independent reconstruction of the linear anisotropic stress $\eta$. J. Cosmol. Astrop. Phys. 11, 027 (2018). arXiv:805.00027

64. R.G. Cai, N. Tamanini, T. Yang, Reconstructing the dark sector interaction with LISA. J. Cosmol. Astrop. Phys. 05, 031 (2017). arXiv: 1703.07323

65. B.S. Haridasu, V.V. Luković, M. Moresco, N. Vittorio, An improved model-independent assessment of the late-time cosmic expansion. J. Cosmol. Astrop. Phys. 10, 015 (2018). arXiv: 1805.03595

66. M.J. Zhang, H. Li, Gaussian processes reconstruction of dark energy from observational data. Eur. Phys. J. C 78, 460 (2018). https://doi.org/10.1140/epjc/s10052-018-5953-3. arXiv: 1806.02981

67. D. Wang, X.H. Meng, Improved constraints on the dark energy equation of state using Gaussian processes. Phys. Rev. D 95, 023508 (2017). arXiv: 1708.07750
68. C.A. Bengaly, C. Clarkson, R. Maartens, The Hubble constant tension with next-generation galaxy surveys. J. Cosmol. Astrop. Phys. 05, 053 (2020). arXiv:1908.04619

69. C.A. Bengaly, Evidence for cosmic acceleration with nextgeneration surveys: A model-independent approach. Mon. Not. Roy. Astron. Soc. 499, L6 (2020). arXiv: 1912.05528

70. R. Arjona, S. Nesseris, What can Machine Learning tell us about the background expansion of the Universe? arXiv:1910.01529

71. R. Sharma, A. Mukherjee, H. Jassal, Reconstruction of late-time cosmology using principal component analysis. arXiv:2004.01393

72. R.C. Nunes, S.K. Yadav, J. Jesus, A. Bernui, Cosmological parameter analyses using transversal BAO data. arXiv:2002.09293

73. K. Liao, A. Shafieloo, R.E. Keeley, E.V. Linder, A modelindependent determination of the Hubble constant from lensed quasars and supernovae using Gaussian process regression. Astrophys. J. Lett. 886, L23 (2019). arXiv:1908.04967

74. A.G. Valent, L. Amendola, H0 from cosmic chronometers and Type Ia supernovae, with Gaussian Processes and the novel Weighted Polynomial Regression methody. J. Cosmol. Astrop. Phys. 04, 051 (2018). arXiv: 1802.01505

75. E. O Colgain and M. M. Sheikh-Jabbari, On model independent cosmic determinations of $H_{0}$. arXiv: 1601.01701

76. R. Briffa, S. Capozziello, J. Levi Said, J. Mifsud, E.N. Saridakis, Constraining Teleparallel Gravity through Gaussian Processes. arXiv:2009.14582

77. C. Krishnan, E. O Colgain, M. M. S. Jabbari, and T. Yang, Running Hubble tension and a $H_{0}$ diagnostic. arXiv:2011.02858

78. M. Moresco et al., A $6 \%$ measurement of the hubble parameter at $z \sim 0.45$ : direct evidence of the epoch of cosmic re-acceleration. J. Cosmol. Astrop. Phys. 05, 014 (2016). arXiv:1601.01701

79. G.-B. Zhao et al., The clustering of the sdss-iv extended baryon oscillation spectroscopic survey dr14 quasar sample: a tomographic measurement of cosmic structure growth and expansion rate based on optimal redshift weights. Mon. Not. Roy. Astron. Soc. 482, 3497 (2019). arXiv:1801.03043

80. H. du Mas des Bourboux et al., The Completed SDSS-IV extended Baryon Oscillation Spectroscopic Survey: Baryon acoustic oscillations with Lyman- $\alpha$ forests. arXiv:2007.08995

81. H. du Mas des Bourboux, Baryon acoustic oscillations from the complete sdss-iii ly $\alpha$-quasar cross-correlation function at $z=2.4$. Astron. Astrophys. 608, A130 (2017). arXiv:1708.02225

82. D. Scolnic et al., The complete light-curve sample of spectroscopically confirmed SNe Ia from Pan-STARRS1 and Cosmological Constraints from the Combined Pantheon Sample. Astrophys. J. 859, 101 (2018). arXiv: 1710.00845

83. A.G. Riess et al., Type Ia supernova distances at redshift $>1.5$ from the Hubble space telescope multi-cycle Treasury Programs: The Early Expansion Rate. Astrophys. J. 853, 126 (2018). arXiv: 1710.00844

84. S. Birrer et al., HOLiCOW - IX. Cosmographic analysis of the doubly imaged quasar SDSS 1206+4332 and a new measurement of the Hubble constant. Mon. Not. Roy. Astron. Soc. 484, 4726 (2019). arXiv: 1809.01274

85. S. Pandey, M. Raveri, B. Jain, Model independent comparison of supernova and strong lensing cosmography: Implications for the Hubble constant tension. Phys. Rev. D 102, 023505 (2020). arXiv: 1912.04325

86. V. Sahni, A. Shafieloo, A.A. Starobinsky, Two new diagnostics of dark energy. Phys. Rev. D 78, 103502 (2008). arXiv:0807.3548

87. A.A. Starobinsky, How to determine an effective potential for a variable cosmological term. JETP Lett. 68, 757 (1998). arXiv:astro-ph/9810431

88. T. Nakamura, T. Chiba, Determining the equation of state of the expanding universe: Inverse problem in cosmology. Mon. Not. Roy. Astron. Soc. 306, 696 (1999). arXiv:astro-ph/9810447 
89. D. Huterer, M.S. Turner, Prospects for probing the dark energy via supernova distance measurements. Phys. Rev. D 60, 081301 (1999). arXiv:astro-ph/9808133

90. T. Abbott et al., Dark energy survey year 1 results: a precise H0 estimate from DES Y1, BAO, and D/H data. Mon. Not. Roy. Astron. Soc. 480, 3879 (2018). arXiv: 1711.00403

91. B. Abbott et al. [LIGO Scientific and Virgo] A gravitational-wave measurement of the Hubble constant following the second observing run of Advanced LIGO and Virgo. arXiv:1908.06060

92. R. Arjona, W. Cardona, S. Nesseris, Unraveling the effective fluid approach for $f(R)$ models in the subhorizon approximation. Phys. Rev. D 99, 043516 (2019). arXiv:1811.02469

93. R. Arjona, W. Cardona, S. Nesseris, Designing Horndeski and the effective fluid approach. Phys. Rev. D 100, 063526 (2019). arXiv: 1904.06294

94. R. Arjona, J. García-Bellido, S. Nesseris, Cosmological constraints on non-adiabatic dark energy perturbations. arXiv:2006.01762

95. W. Cardona, L. Hollenstein, M. Kunz, The traces of anisotropic dark energy in light of Planck. J. Cosmol. Astrop. Phys. 07, 032 (2014). arXiv: 1402.5993

96. F. Piazza, S. Tsujikawa, Dilatonic ghost condensate as dark energy. J. Cosmol. Astrop. Phys. 07, 004 (2004). arXiv:hep-th/0405054

97. R.J. Scherrer, Purely kinetic k-essence as unified dark matter. Phys. Rev. Lett. 93, 011301 (2004). arXiv:astro-ph/0402316

98. G.W. Horndeski, Second-order scalar-tensor field equations in a four-dimensional space. Int. J. Theor. Phys. 10, 363 (1974)

99. C. Deffayet, X. Gao, D.A. Steer, G. Zahariade, From k-essence to generalised Galileons. Phys. Rev. D 84, 064039 (2011). arXiv: 1103.3260

100. T. Kobayashi, M. Yamaguchi, J. Yokoyama, Generalized Ginflation: Inflation with the most general second-order field equations. Prog. Theor. Phys. 126, 511 (2011). arXiv:1105.5723

101. E. Bellini, I. Sawicki, Maximal freedom at minimum cost: linear large-scale structure in general modifications of gravity. J. Cosmol. Astrop. Phys. 07, 050 (2014). arXiv: 1404.3713

102. The LIGO Scientific Collaboration, the Virgo Collaboration, GW170817: observation of gravitational waves from a binary neutron star inspiral. Phys. Rev. Lett. 119, 161101 (2017). arXiv: 1710.05832

103. LIGO Scientific Collaboration, Virgo Collaboration, Fermi Gamma-Ray Burst Monitor, INTEGRAL, Gravitational Waves and Gamma-rays from a Binary Neutron Star Merger: GW170817 and GRB 170817A. Astrophys. J. Lett. 848, L13 (2017). arXiv: 1710.05834
104. T. Delubac et al., Baryon acoustic oscillations in the $L y \alpha$ forest of BOSS DR11 quasars. Astron. Astrophys. 574, A59 (2015). arXiv: 1404.1801

105. É. Aubourg et al., [BOSS], Cosmological implications of baryon acoustic oscillation measurements. Phys. Rev. D 92, 123516 (2015). arXiv:1411.1074

106. B. Boisseau, G. Esposito-Farese, D. Polarski, A.A. Starobinsky, Reconstruction of a scalar tensor theory of gravity in an accelerating universe. Phys. Rev. Lett. 85, 2236 (2000). arXiv:gr-gc/0001066

107. V. Sahni, A. Starobinsky, Reconstructing dark energy. Int. J. Mod. Phys. D 15, 2105 (2006). arXiv:astro-ph/0610026

108. A.D. Dolgov, Field model with a dynamic cancellation of the cosmological constant. JETP Lett. 41, 345 (1985)

109. P. Brax, C. van de Bruck, Cosmology and brane worlds: a review. Class. Quant. Grav. 20, R201 (2003). arXiv:hep-th/0303095

110. Ö. Akarsu, N. Katırc1, S. Kumar, Cosmic acceleration in a dust only universe via energy-momentum powered gravity. Phys. Rev. D 97, 024011 (2018). arXiv: 1709.02367

111. O. Akarsu, N. Katirci, S. Kumar, R. C. Nunes, B. Ozturk, S. Sharma, Rastall gravity extension of the standard $\Lambda \mathrm{CDM}$ model: theoretical features and observational constraints. arXiv:2004.04074

112. Ö. Akarsu, J.D. Barrow, L.A. Escamilla, J.A. Vazquez, Graduated dark energy: Observational hints of a spontaneous sign switch in the cosmological constant. Phys. Rev. D 101, 063528 (2020). arXiv: 1912.08751

113. L. Visinelli, S. Vagnozzi, U. Danielsson, Revisiting a negative cosmological constant from low-redshift data. Symmetry 11, 1035 (2019). arXiv: 1907.07953

114. C.E. Rasmussen, C.K.I. Williams, Gaussian processes for machine learning (MIT Press, London, 2006). ISBN 0-26218253-X

115. F. Renzi, A. Silvestri, A look at the Hubble speed from first principles. arXiv:2011.10559

116. S. Alam, et al. (eBOSS Collaboration), The Completed SDSS-IV extended Baryon Oscillation Spectroscopic Survey: Cosmological Implications from two Decades of Spectroscopic Surveys at the Apache Point observatory. arXiv:2007.08991

117. O.H.E. Philcox, M.M. Ivanov, M. Simonovic, M. Zaldarriaga, Combining full-shape and $\mathrm{BAO}$ analyses of galaxy power spectra: a 1.6\% CMB-independent constraint on H0. J. Cosmol. Astrop. Phys. 05, 032 (2020). arXiv:2002.04035

118. T.M.C. Abbott et al., (DES Collaboration), Dark Energy Survey Year 1 Results: A Precise H0 Measurement from DES Y1, BAO, and D/H Data. Mon. Not. Roy. Astron. Soc. 480, 3 (2018). arXiv: 1711.00403 